\title{
VAR Modeling for Dynamic Semiparametric Factors of Volatility Strings
}

\author{
Ralf Brüggemann* \\ Wolfgang Härdle* \\ Julius Mungo* \\ Carsten Trenkler*
}

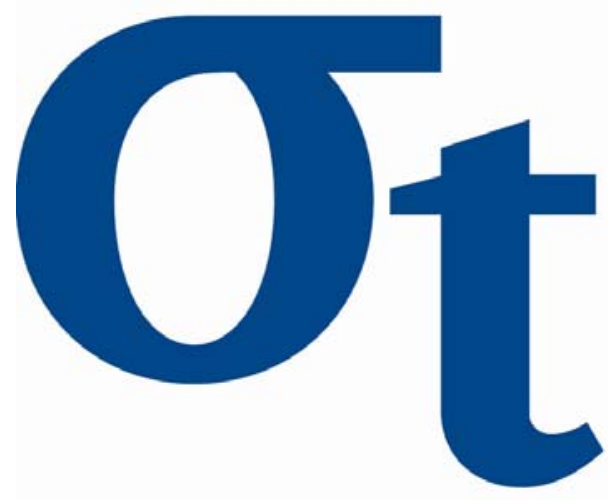

* Institute of Statistics and Econometrics and CASE - Center for Applied Statistics and Economics, Humboldt-Universität zu Berlin, Germany

This research was supported by the Deutsche Forschungsgemeinschaft through the SFB 649 "Economic Risk". 


\title{
VAR Modeling for Dynamic Semiparametric Factors of Volatility Strings *
}

\author{
Ralf Brüggemann Wolfgang Härdle Julius Mungo \\ Carsten Trenkler
}

Humboldt-Universität zu Berlin

Department of Economics

Spandauer Str. 1

D-10178 Berlin

February 3, 2006

\begin{abstract}
The implied volatility of a European option as a function of strike price and time to maturity forms a volatility surface. Traders price according to the dynamics of this high dimensional surface. Recent developments that employ semiparametric models approximate the implied volatility surface (IVS) in a finite dimensional function space, allowing for a low dimensional factor representation of these dynamics. This paper presents an investigation into the stochastic properties of the factor loading times series using the vector autoregressive (VAR) framework and analyzes associated movements of these factors with movements in some macroeconomic variables of the Euro economy.
\end{abstract}

Keywords: Implied volatility surface, dynamic semiparametric factor model, unit root tests, vector autoregression, impulse responses.

JEL classification: C14; C32

*This research was supported by the Deutsche Forschungsgemeinschaft through the SFB 649 'Economic Risk'. Corresponding author: Julius Mungo, Center for Applied Statistics and Economics, HumboldtUniversität zu Berlin, Department of Economics, Spandauer Str. 1, D-10178 Berlin, Germany, email: mungo@wiwi.hu-berlin.de 


\section{Introduction}

The valuation of options has become increasingly important with the arrival of new financial products. Financial instruments with optional features such as standard options, derivatives like weather options or exotic types like Down-and-out Puts are increasingly being traded on the markets. A substantial part of this option trading focuses on market volatility (Chaput and Ederington, 2002). Since volatility is unknown, one studies the implied volatility (IV) which is derived from the Black-Scholes formula (e.g. Black and Scholes, 1973 and Franke, Härdle and Hafner, 2004) for a cross section of options with different strike and maturities traded at the same point in time. The implied volatility depends on the strike and maturity and is therefore better described as an implied volatility surface (IVS). The movement of this surface may be summarized by a dynamic semiparametric factor model (DSFM) as developed by Fengler et al. (2005). Their model is based on the following additive structure for the log-implied volatility, $Y_{t, j}$ :

$$
Y_{t, j}=\sum_{k=0}^{K} z_{t k} m_{k}\left(X_{t, j}\right)+\varepsilon_{t, j}
$$

where $z_{t 0}=1, j=1, \ldots, J_{t}$ represents the number of IV observations on day $t$ and $K$ is the number of basis functions. The $X_{t, j}$ is a two-dimensional variable containing moneyness and maturity. The $z_{t k}$ are time dependent weights of the smooth basis function $m_{k}$, for $(k=0, \ldots, K)$. Models similar to (1.1) have been considered by Skiadopoulos, Hodges and Clewlow (1999) in studies exploring the volatility smile in different maturity buckets and Alexander (2001) in analyzing fixed strike deviations.

The IVS is assumed to be a weighted sum of the functional factors and the dynamics is explained by the stochastic behavior of the loadings. The estimates $\widehat{z}_{t k}$ and $\widehat{m}_{k}$ are obtained by minimizing the following least squares criterion $\left(z_{t 0}=1\right)$ :

$$
\sum_{t=1}^{I} \sum_{j=1}^{J_{t}} \int\left\{Y_{t, j}-\sum_{k=0}^{K} z_{t k} m_{k}(u)\right\}^{2} K_{h}\left(u-X_{t, j}\right) d u
$$

where $K_{h}$ denotes a two-dimension kernel function, chosen as a product of one-dimensional kernels $K_{h}(u)=k_{h_{1}}\left(u_{1}\right) \times k_{h_{2}}\left(u_{2}\right)$, where $h=\left(h_{1}, h_{2}\right)^{\top}$ are bandwidths and $k_{h}(v)=k\left(h^{-1} v\right) / h$ is a one-dimensional kernel function. 
The minimization procedure searches through all functions $\widehat{m}_{k}: \mathbb{R}^{2} \longrightarrow \mathbb{R}(k=0, \ldots, K)$ and time series $\widehat{z}_{t k} \in \mathbb{R}(t=1, \ldots, I ; k=1, \ldots, K)$. Borak et al. (2005) presented a DSFM for DAX option data for a period from January 4, 1999 to February 25, 2003. Their data consists of $J_{t} \approx 5000$ observations per day and the model captures the complex dynamic structure with a low dimensional representation of the IVS, i.e. the IVS is approximated by a small number of basis functions in a finite dimensional space. A typical shape of an IVS surface, considered as a function of time to maturity and moneyness (e.g. Borak et al., 2005) is presented in Figure 1. This figure shows the IVS for the DAX trade on May 2, 2000 using a semiparametric factor model fit. As time changes the IVS moves in space and time. Recent research has been geared towards analyzing the behavior of the IVS as an important element of prediction. Skiadopoulos, Hodges and Clewlow (1999) have analyzed the IVS of S\&P 500 options for the years 1992-1995 and reported that at least two and at most six factors are necessary to capture the dynamics of S\&P 500 implied volatility. Cont and Fonseca (2002) worked on the dynamics of the S\&P 500 implied volatility and reported that the first three principal components account for $95 \%$ of the daily variance. Based on the closing prices of DAX options during the year 1999, Fengler, Härdle and Villa (2003) concluded that three factors are sufficient to capture $95 \%$ of the variation in implied DAX volatilities. In the DSFM, the movement of the IVS can be described (see Borak et al.,2005) by the loading series $z_{t}=\left(z_{t 1}, \ldots, z_{t K}\right)^{\top}$, which are obtained after fitting a model like in (1.1). Their model is obtained by setting $K=3$ such that three basis functions are used to model implied volatility. In this paper we examine the stochastic properties of the corresponding factor loading times series from Borak et al., 2005 by using vector autoregressive (VAR) modeling techniques.

This modeling framework is fairly general in describing the dynamics and interrelations between the variables of interest. The factor loadings describe the movements of the implied volatility surface such that $z_{1}$ may be interpreted as representing the overall shift volatility (or trend), $z_{2}$ represents changes in the maturity slope while $z_{3}$ represents changes in the smile curvature (moneyness slope) of the IVS. 
Therefore, understanding the dynamics of $z_{t}$ may provide insights in the behavior of the IVS, which in turn helps to give an accurate assessment of market risk. In addition, understanding the IVS dynamics is also important for developing useful strategies to hedge against these risks. To provide further insight into factors that influence the IVS behavior, we extend our investigation by associating movements of these risk factors with movements in some macroeconomic conditions. The paper is organized as follows. We briefly describe the data used in our study and analyze the order of integration of the factor loading series using a number of unit root tests in Section 2. Section 3 presents results from the VAR modeling and describes the dynamic interaction between the factor loading series. In section 4 we relate these risk factors to macroeconomic indicators such as interest rates, exchange rates and oil prices. We conclude with an interpretation of results in Section 5.

\section{The Data and Unit Root Tests}

We analyze time series data on factor loading series that have been obtained from a DSFM model specified by Borak et al. (2005). The DSFM summarizes the IVS dynamics by $K=3$ basis functions. Accordingly, we analyze three loading series $z_{t}=\left(z_{t 1}, z_{t 2}, z_{t 3}\right)^{\top}$ for a period from January 4, 1999 to February 25, 2003. Excluding days with no option trades we have $T=1052$ observations on $z_{t}$ in our sample. Corresponding time series plots are given in the left column of Figure 2, while plots for the first differences, $\Delta z_{t}=z_{t}-z_{t-1}$, are given in the right column of the Figure.

The level series show volatility characteristics as they are often found for financial market data. We observe strong day-to-day variation, especially in the first loading series and a number of volatility clusters. Furthermore, there are indications of structural breaks in the series. To be precise, we observe a sudden downward movement in $z_{t 1}$ in September 2001, $z_{t 2}$ exhibits a clear outlier in November 2001, which is also seen in the first differences and the third loading series $z_{t 3}$ shows much stronger volatility in the first part of the sample than in the second one. In addition, the graphs of $z_{t 3}$ and its first differences also point towards a number of possible outliers. 
As a first step in analyzing the statistical properties, we test whether the series are integrated of order zero $(I(0))$ or of order one $(I(1))$. The order of integration is important for the further modeling strategy. In case of stationarity $(I(0))$ we proceed with analyzing a VAR model for the level series. By contrast, if the series are $I(1)$, a VAR model in first differences would be a more appropriate choice. In the following we apply the ADF and ERS unit root tests. The ADF test refers to the regression equation

$$
\Delta z_{t, k}=\phi z_{t-1, k}+\sum_{i=1}^{p} \alpha_{i} \Delta z_{t-i, k}+u_{t, k}
$$

where $p$ is the number of lags of $\Delta z_{t, k}$ by which the regression equation (2.1) is augmented in order to get residuals free of autocorrelation.

Under the null hypothesis of a unit root the parameter $\phi$ should be zero. Hence, the $t$-statistic of the OLS estimator of $\phi$ is used as the ADF test statistic. Note that the limiting distribution of the test statistic is nonstandard. Hence, critical or $p$-values have to be derived by the help of simulation methods.

The lag order $p$ is determined by applying the AIC, HQ, and SC information criteria. ${ }^{1}$ Since the test decisions may depend on the suggested order we will present our results for AIC and HQ. The SC criterion tends to propose orders which are too low to capture the autocorrelation contained in the factor loading series.

Since the ADF test suffers from low power and therefore may fail to detect a stationary time series we also consider the point-optimal unit root test (ERS test) suggested by Elliot, Rothenberg and Stock (1996). The small sample simulation results of Elliot et al. (1996) indicate that this test is superior to the ADF procedure also in case of processes affected by conditional heteroscedasticity. The test is based on quasi-differences of $z_{t, k}$ which are defined by

$$
d\left(z_{t, k} \mid a\right)= \begin{cases}1 & \text { if } t=1 \\ z_{t, k}-a z_{t-1, k} & \text { if } t>1\end{cases}
$$

where $a$ represents the point alternative against which the null of a unit root is tested. We follow the suggestion of Elliot et al. (1996) and use $a=\bar{a}=1-7 / T$ since only a constant term is considered.

\footnotetext{
${ }^{1}$ The criteria are described in Lütkepohl (1991).
} 
Let $\hat{e}_{t}$ be the residuals from a regression of the time series on a quasi-differenced constant and let $S(\bar{a})$ and $S(1)$ be the sums of squared residuals for the cases $a=\bar{a}$ and $a=1$ respectively. Then the test is defined by

$$
E R S=\{S(a)-a S(1)\} / \hat{\omega}_{b}
$$

where $\hat{\omega}_{b}$ is the spectral density estimator of $\hat{e}_{t}$ at frequency zero. We apply the autoregressive spectral density estimator as proposed by Elliot et al. (1996). In order to determine the lag length $b$ of the corresponding estimation regressions the AIC and HQ criteria are used. The limiting distribution of the test statistic is nonstandard and critical values are stated in Elliot et al. (1996).

Although the tests' results do not agree in all cases, they suggest that the three loading series can be regarded as stationary. In other words, the null hypothesis of a unit root is usually rejected for the level series. However, the series exhibit possible structural breaks. As mentioned above, the series $z_{t 2}$ contains a large outlier in November 2001 and the fluctuations of $z_{t 3}$ seem to be much weaker in the second half of the sample than in the first one. Hence, size distortions may occur. To account for possible structural breaks we have also performed a unit root analysis for the two subsamples January 4, 1999-July 31, 2001 (655 observations) and August 1, 2001- February 25, 2003 (397 observations). The results for the subsamples confirm our findings for the whole data period regarding $z_{t 2}$ and $z_{t 3}$. By contrast, the tests for the subperiods seem to indicate nonstationarity of $z_{t 1}$. Summarizing, the unit root analysis suggests stationarity of loading series. However, some disagreements have occurred. Therefore, we proceed to work with a VAR model for the levels of the series in order to avoid possible overdifferencing and check the robustness of our results by also analyzing a VAR model in first differences. 


\section{VAR Models for Factor Loading Dynamics}

VAR models are often used to investigate the dynamic relationship between the variables of interests. In our case, we use the VAR modeling framework to investigate the relationship between the factor loading times series described earlier.

As the results of the unit root analysis are not clear cut, we use both a VAR in levels and a VAR in first differences to model the interdependencies between the factor loadings. As before $z_{t}=\left(z_{1 t}, z_{2 t}, z_{3 t}\right)^{\top}$ denotes the vector containing the observations of the $K=3$ factor loadings $z_{1 t}, z_{2 t}$ and $z_{3 t}$ at time $t$. Then, we model the dynamics underlying these factor loading by a VAR process of order $p, \operatorname{VAR}(p)$,

$$
z_{t}=\nu+A_{1} z_{t-1}+\cdots+A_{p} z_{t-p}+u_{t}
$$

where $\nu$ is a $K \times 1$ vector of intercept parameters, $A_{i}, i=1, \ldots, p$ are $K \times K$ parameter matrices and $u_{t}=\left(u_{1 t}, \ldots, u_{K t}\right)^{\top}$, is an unobservable error term with mean zero and time-invariant, non-singular covariance matrix $\Sigma_{u}$, i.e. $\mathrm{E}\left[u_{t} u_{t}^{\top}\right]=\Sigma_{u}$.

Given the integration properties of the underlying time series discussed in Section 2 an alternative way of modeling the factor loadings $z_{t}$ is to specify a VAR model in first differences, i.e. a model for $\Delta z_{t}=z_{t}-z_{t-1}$ is considered. The corresponding VAR is of the form

$$
\Delta z_{t}=\nu+A_{1} \Delta z_{t-1}+\cdots+\Delta A_{p} z_{t-p}+u_{t}
$$

We start our empirical analysis by considering data for the whole sample period from January 4, 1999 until February 25, 2003. Moreover, we focus first on a model for the levels of our variables, i.e. on the model for $z_{t}$ given in (3.1). To select the lag length $p$, we have applied standard information criteria as in Lütkepohl (1991, Chapter 4) to VAR models with a maximum lag order of $p_{\max }=12$ and give the results in Table 2 . The three considered information criteria suggest different lag lengths for the sample under consideration. As we want to model the dynamic relations between the factor loading series by impulse response functions we start out by a fairly general model with $p=7$ lags as suggested by the Akaike information criterion (AIC). 
Choosing the lag length by AIC may be advantageous for our purpose given the results in Brüggemann (2004), as a fairly large model allows to capture the underlying dynamics in a more flexible way. We check the adequacy of the model by applying a number of standard diagnostic tests whose results are reported in Table 3. While the estimated residuals do not show signs of autocorrelation, normality and conditional homoscedasticity are clearly rejected. Both, non-normality and ARCH effects are often observed in empirical models for financial variables. In fact, given the time series plots in Figure 2, these results are not surprising. In what follows, we ignore these short-coming of our model ${ }^{2}$ and regard the VAR model as a rough approximation of the underlying DGP.

The dynamic relations between the factors is captured by the impulse response function, Lütkepohl (1991, Chapter 3) which we discuss next. Figure 3 shows the impulse responses together with \pm 2 asymptotic standard error bands from the estimated model for $z_{t}$, where the shocks have been orthogonalized by a Cholesky decomposition.

In the first column of the figure, we give the responses to a positive innovation in the first loading series. This innovation has a permanent negative effect on the second factor loading $z_{t 2}$ and a small but positive effect on $z_{t 3}$, which becomes insignificant after about 6 periods. In contrast, an innovation in the second loading factor (column 2 of Figure 3) has only a permanent positive effect on $z_{t 2}$, while the effects on the other variables are not significantly different from zero. A similar result is obtained for a shock in the third loading series. While it has a permanent positive effect on itself, no significant responses on the other variables are observed. Clearly, the results of the impulse response analysis may depend to some extent on the ordering of the variables in the system. In fact, the estimated residual correlation matrix for our benchmark model is

$$
\hat{P}_{u}=\left(\begin{array}{rrr}
1 & -0.49 & -0.23 \\
-0.49 & 1 & -0.10 \\
-0.23 & -0.10 & 1
\end{array}\right) \text {. }
$$

Given the sample size of $T=1052$ observations, the off-diagonal elements of $\hat{P}_{u}$ are fairly large and changing the ordering of the variables may indeed change the results from the impulse-response analysis.

\footnotetext{
${ }^{2} \mathrm{~A}$ more detailed analysis of the ARCH structure is beyond the scope of this paper.
} 
We have tried all possible variable orderings when computing the impulse responses and the only notable change is related to the response of $z_{t 1}$ to an innovation in $z_{t 2}$. For instance, when the ordering $z_{t}=\left(z_{t 2}, z_{t 1}, z_{t 3}\right)^{\top}$ is considered, we find a permanent, significant negative response of $z_{t 1}$. Independent of the orderings, we present in Figure 4 the generalized impulse responses that account for the residual correlation (Pesaran and Shin, 1998). In comparison, the main dynamics in Figure 3 and Figure 4 are similar. According to our analysis there is quite a bit of interaction between $z_{t 1}$ and $z_{t 2}$. The only change is related to the long-run significance of the response in $z_{1 t}$ to a shock in $z_{2 t}$ and vice versa. The full sample period includes days with events that led to turbulence on stock markets. The most obvious is the terrorism attack of September 11, 2001. The events may be viewed as structural breaks that possibly affect the parameter of our model. Therefore, we check the robustness of our results by considering a sub-sample that uses data until July 31, 2001 only. For this sub-sample the AIC suggest a lag length of $p=3$ (see Table 2) but checking the diagnostic tests (see Table 3) reveals that this lag specification is not sufficient to render the residuals free of autocorrelation. However, increasing the lag length for the sub-sample to $p=8$ leads to residuals that do not show signs of autocorrelation. As in the model for the full sample, there is evidence for non-normality and $\mathrm{ARCH}$ in the residuals. Another robustness check is related to the model specification of the VAR model. In addition to the results based on a model for $z_{t}$ we have also obtained results for a model for the first differences of $z_{t}$, i.e. we consider the model for $\Delta z_{t}$ given in (3.2). The relevant statistics are given in Table 2 and 3. For the full sample, we use a VAR(6) and for comparison with the levels model, we analyze the accumulated impulse responses from a model for $\Delta z_{t}$. In comparison to Figure 3 the results are virtually identical to the results from the model for $z_{t}{ }^{3}$

\footnotetext{
${ }^{3} \mathrm{~A}$ comparison of the sub-sample model leads to the same conclusions. Therefore, the results are not reported here to conserve space.
} 
To further investigate the relationship between the variables we have also conducted Granger causality tests as well as tests for instantaneous causality (see e.g. Granger, 1969 and Lütkepohl, 1991, Chapter 2). The results of the tests are given in Table $4 .{ }^{4}$ Granger non-causality of $z_{1}$ for $z_{2}$ and $z_{3}$ and of $z_{2}$ for $z_{1}$ and $z_{3}$ is rejected at least at the $10 \%$ significance level (see top panel in Table 4). Investigating the causal relationships more closely reveals that $z_{3}$ is neither Granger-caused by $z_{1}$ nor $z_{2}$. Moreover, Granger non-causality from $z_{1}$ to $z_{3}$ and from $z_{2}$ to $z_{3}$ cannot be rejected. In other words, including $z_{3}$ does not help predicting $z_{1}$ and $z_{2}$ and predictions of $z_{3}$ are not improved by including $z_{1}$ and $z_{2}$. This result is in line with the impulse response pattern discussed earlier. A possible conclusion is that $z_{3}$ may as well be excluded from the VAR system. In fact, using any of the sequential model reduction algorithms discussed in Brüggemann (2004, Chapter 2) leads to a model where lags of $z_{3}$ are excluded from the equations for $z_{1}$ and $z_{2}$ and moreover, $z_{3}$ only depends on its own lags. This result confirms the finding of the Granger causality tests. ${ }^{5}$ Contemporaneous non-causality is rejected for all variables in the system, which is inline with the relative large residual correlations given in equation (3.3). To sum up, the VAR model analysis has revealed that there is a quit bit of interaction between the first and second loading. A positive shock in the first loading has a permanent negative impact on the second and vice versa. Note that movements in $z_{1}$ may be interpreted as overall shifts (up or down) of the IVS whereas $z_{2}$ represents changes in the maturity slope of the IVS. Thus, an overall increase in financial market risk is associated with an upward tilt of the maturity slope. In other words, the risk of longer maturities increases relative to shorter maturities. Accordingly, a decrease in the relative risk of long-term options induces a general risk reduction (lower overall implied volatility). Moreover, we find that $z_{1}$ and $z_{2}$ Granger-cause each other. By contrast, the third loading series is not importantly related to $z_{1}$ and $z_{2}$. Hence, changes in the moneyness slope of the IVS are not directly linked to up- or downward shifts of the IVS or to adjustments in the relative risk of long- and short-term options.

\footnotetext{
${ }^{4}$ Testing Granger causality involves testing zero restrictions of some VAR coefficients, which may have a non-standard asymptotic distribution when $I(1)$ variables are in the system. To overcome this problem, Toda and Yamamoto (1995) and Dolado and Lütkepohl (1996) suggest to overfit the VAR model by one lag to remove the singularity of the coefficient covariance matrix. Applying this procedure leads to identical conclusions.

${ }^{5}$ We have repeated the impulse response analysis for a system including only $z_{1}$ and $z_{2}$ and found impulseresponses virtually identical to the upper left $2 \times 2$ block of Figure 4 .
} 
Moreover, we find that neither the first nor the second factor have a strong influence on the third. In other words, a VAR model for the first two factors only would capture the important dynamics between the factor loadings. This result has also been confirmed by using Granger causality tests and model reduction procedures. As robustness check we have also considered models in first differences and models for subsamples and find similar results for all model specifications.

\section{Loadings and macroeconomic indicators}

In this section we extend our benchmark specification VAR model to include some macroeconomic time series namely, the log of US dollar per Euro (LEX), the log of oil prices (LPOIL) and the 12-months German money market interest rate (R12M) from 1.04.1999 - 2.25.2003, (see Figure 5). A VAR(8) is analyzed to be the best choice for the system $z_{t}=\left(z_{1 t}, z_{2 t}, z_{3 t}, \mathrm{LEX}, \mathrm{LPOIL}, \mathrm{R} 12 \mathrm{M}\right)^{\top}$. The dynamic characteristics of the system is exploited through the generalized impulse response technique (Pesaran and Shin, 1998) and the results are presented in Figures 6 and 7. There are a number of significant impulse responses. In Figure 6, the 12-month interest rate responds significantly to shocks in all three loading series in a permanent way. A positive shock in $z_{1 t}$ represents a general increase in volatility, i.e. a higher overall risk. A rise in $z_{2 t}$ means that the maturity slope of the implied vola surface is tilted downwards. Thus, the risk of longer maturities decreases relative to shorter maturities. Moreover, a shock to $z_{3 t}$ affects the moneyness slope and smile curvature (Borak et al., 2005). Thus, the responses of R12M to shocks in the loading series may be regarded as changes in risk compensation. Accordingly, higher overall risk induces an interest rate rise, whereas lower relative risk of longer maturities requires reduced risk compensation in terms of the 12-month interest rate. Note that we consider maturities of up to six months. Finally, a positive shock to $z_{3 t}$ enhances the smile curvature and raises the relative risk of options with low moneyness value. This creates a permanent positive response in $\mathrm{R} 12 \mathrm{M}$ or, in other words, induces a need for higher risk compensation at the 12-month horizon. 
Interestingly, we also observe in Figure 7 that $z_{1 t}$ and $z_{2 t}$ significantly respond to a shock in $\mathrm{R} 12 \mathrm{M}$. If we regard rising interest rates as an indicator for higher inflation and worsening economic prospects then it is likely that higher interest rates are accompanied by increasing uncertainty in the financial markets. This pushes the first loading upward meaning higher overall volatility or risk in the financial markets. Furthermore, the relative risk of options with longer maturities goes up. This is shown by the negative response of $z_{2 t}$. Since the third loading does not respond significantly to changes in the interest rate (macro)economic affects seem to feed into financial market risks via the maturity channel rather than via the moneyness dimension. With respect to the other two economic variables only the exchange rate has some relevance in the way it is significantly related to the first loading. A positive shock to LEX leads to a negative response in $z_{1 t}$ and vice versa and the responses are significant for up to 20 days. Given our empirical results a rise in LEX, i.e. an appreciation of the Euro, reduces the volatility of the DAX-Options. Hence, a stronger Euro translates to a lower risk in the German stock market. Similarly, higher financial market risk (positive shock in $z_{1 t}$ ) induces an depreciation of the Euro with respect to the US dollar.

\section{Conclusions}

In this paper, we have analyzed and modeled the stochastic properties of factors of Volatility Strings derived from a dynamic semiparametric model for implied volatility. The VAR modeling framework applied provides a fairly good description of the dynamics and interrelations between the factor loadings that determine the movements of the IVS. The relationship of the loadings to movements in macroeconomic variables (interest rates, exchange rates, oil prices) of the Euro-economy was also investigated. Our results reveal that only the 12-month interest rate is importantly related to the factor loading series. Since interest rates at the one-year horizon reflect expectations about future economic developments, these links seem to be very important for our understanding of the relationship between financial market and macroeconomic risk. A closer inspection of interest rates for other horizons may be very promising. 
In contrast, oil prices are not very important for the loadings driving the implied volatility surface. However, the Dollar-Euro exchange is significantly linked to the overall risk in the German stock market. This study may be seen as a first step in associating movements in some risk factors with movements in macroeconomic conditions, an ingredient necessary to give an accurate assessment of market risks. Therefore, an important outlook is then to develop useful strategies for hedging against these risk factors. 


\section{References}

1. Alexander, C., 2001, Principles of the skew, Risk 14 (1), 29-32.

2. Black, F. and M. Scholes, 1973, The pricing of options and corporate liabilities, Journal of Political Economy 81, 637-659.

3. Borak, S., W. Härdle and M. Fengler, 2005, DSFM fitting of Implied Volatility Surfaces, Proceedings 5th International Conference on Intelligent, System Design and Applications, IEEE Computer Society Number P2286, Library of Congress Number 2005930524.

4. Brüggemann, R., 2004, Model Reduction Methods for Vector Autoregressive Processes, Vol. 536 (Lecture Notes in Economics and Mathematical Systems), Berlin: Springer-Verlag.

5. Chaput, J.S. and L.H. Ederington, 2002, Volatility trade design, Working paper, University of Otago.

6. Cont, R. and J.D. Fonseca, 2002, The Dynamics of Implied Volatility Surfaces, Quantitative Finance 2(1), 45-602.

7. Dolado, J. J. and H. Lütkepohl, 1996, Making Wald tests work for cointegrated VAR systems, Econometric Reviews 15, 369-386.

8. Doornik, J. A. and H. Hansen, 1994, A practical test of multivariate normality, unpublished paper, Nuffield College.

9. Elliot, G., T. J. Rothenberg and J. H. Stock, 1996, Efficient tests for an autoregressive unit root, Econometrica 64, 813-836.

10. Fengler, M., 2005, Semiparametric Modeling of Implied Volatility, Springer-Verlag. 
11. Fengler, M., W. Härdle and E. Mammen, 2005, A dynamic semiparametric factor model for implied volatility string dynamics, SFB 649, Discussion paper, 2005-020, Humboldt-Universität zu Berlin.

12. Fengler, M., W. Härdle and C. Villa, 2003, The dynamics of implied volatilities: A common principal component approach, Review of Derivatives Research 6, 197-202.

13. Franke, J., W. Härdle and M.C. Hafner, 2004, Statistics of Financial Markets, Springer-Verlag.

14. Granger, C. W. J., 1969, Investigating causal relations by econometric models and cross-spectral methods, Econometrica 37, 424-438.

15. Kwiatkowski, D., P.C.B. Phillips, P. Schmidt and Y. Shin, 1992, Testing the null hypothesis of stationarity against the alternative of a unit root, Journal of Econometrics 54, 159-178.

16. Lütkepohl, H., 1991, Introduction to Multiple Time Series Analysis, Berlin: Springer-Verlag.

17. Lütkepohl, H., 2004, Vector autoregressive and vector error correction models, in H. Lütkepohl and M. Krätzig (eds), Applied Time Series Econometrics, Cambridge University Press, Cambridge.

18. Lütkepohl, H. and M. Krätzig, (eds) 2004, Applied Time Series Econometrics, Cambridge University Press, Cambridge.

19. MacKinnon, J. G., 1991, Critical values for cointegration tests, in C.W.J. Granger and R.F. Engle (eds), Long-Run Economic Relationships, Oxford University Press, Oxford.

20. Newey, W. and K. West, 1994, Automatic lag selection in covariance matrix estimation, Review of Economic Studies 61, 631-653. 
21. Pesaran, M.H. and Y. Shin, 1998, Generalized impulse response analysis in linear multivariate models, Economics Letters 58, 17-29.

22. Skaidopoulos, G., S. Hodges and L. Clewlow, 1999, The dynamics of S\&P 500 implied volatility surface, Review of Derivatives Research 3, 263-282.

23. Toda, H. Y. and T. Yamamoto, 1995, Statistical inference in vector autoregressions with possibly integrated processes, Journal of Econometrics 66, 225-250. 


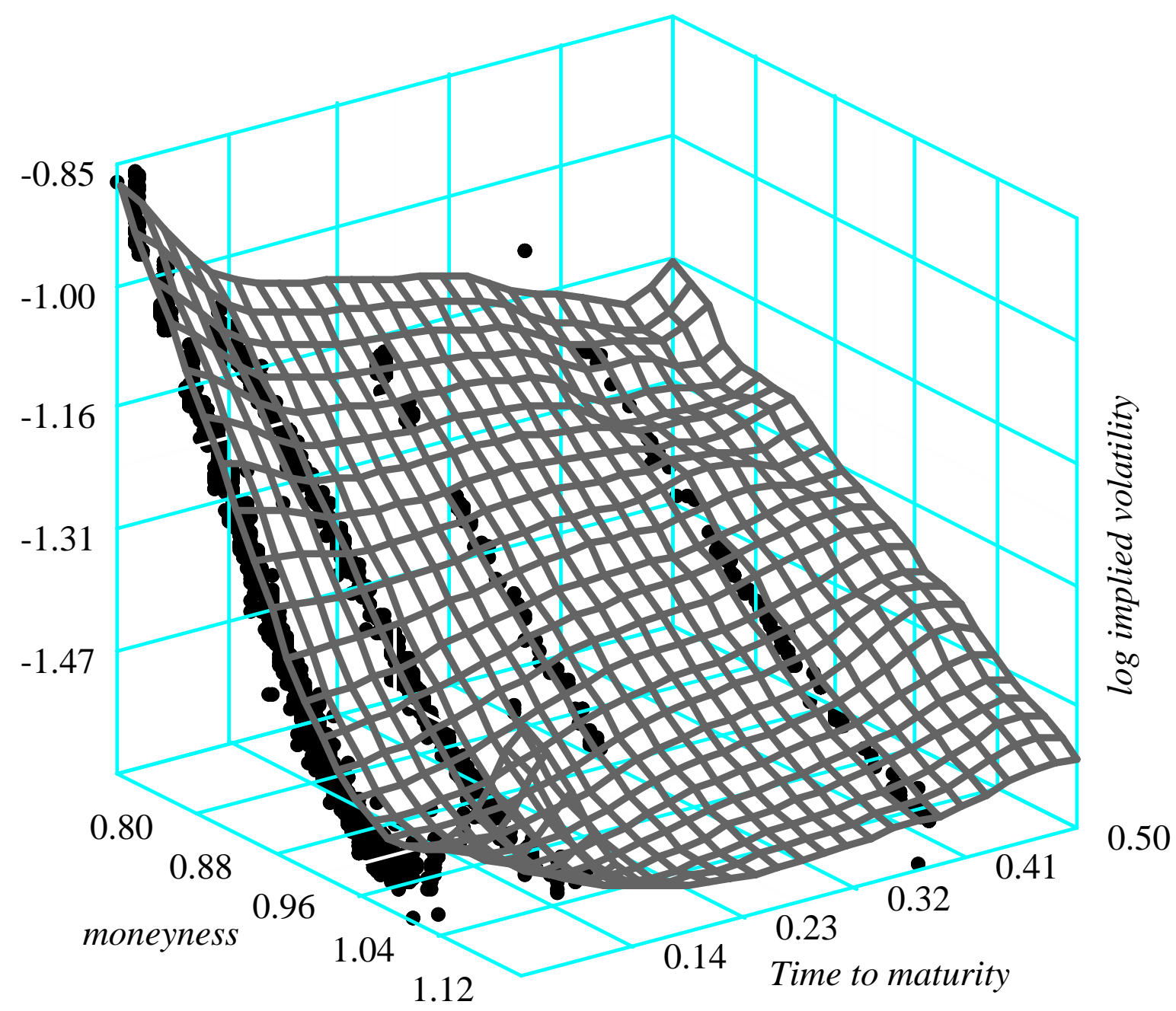

Figure 1: Implied volatility surface from DSFM fit for the DAX-Option on 2 May 2000, with moneyness between 0.8 and 1.12 and time to maturity between 0 and 0.5 years. 

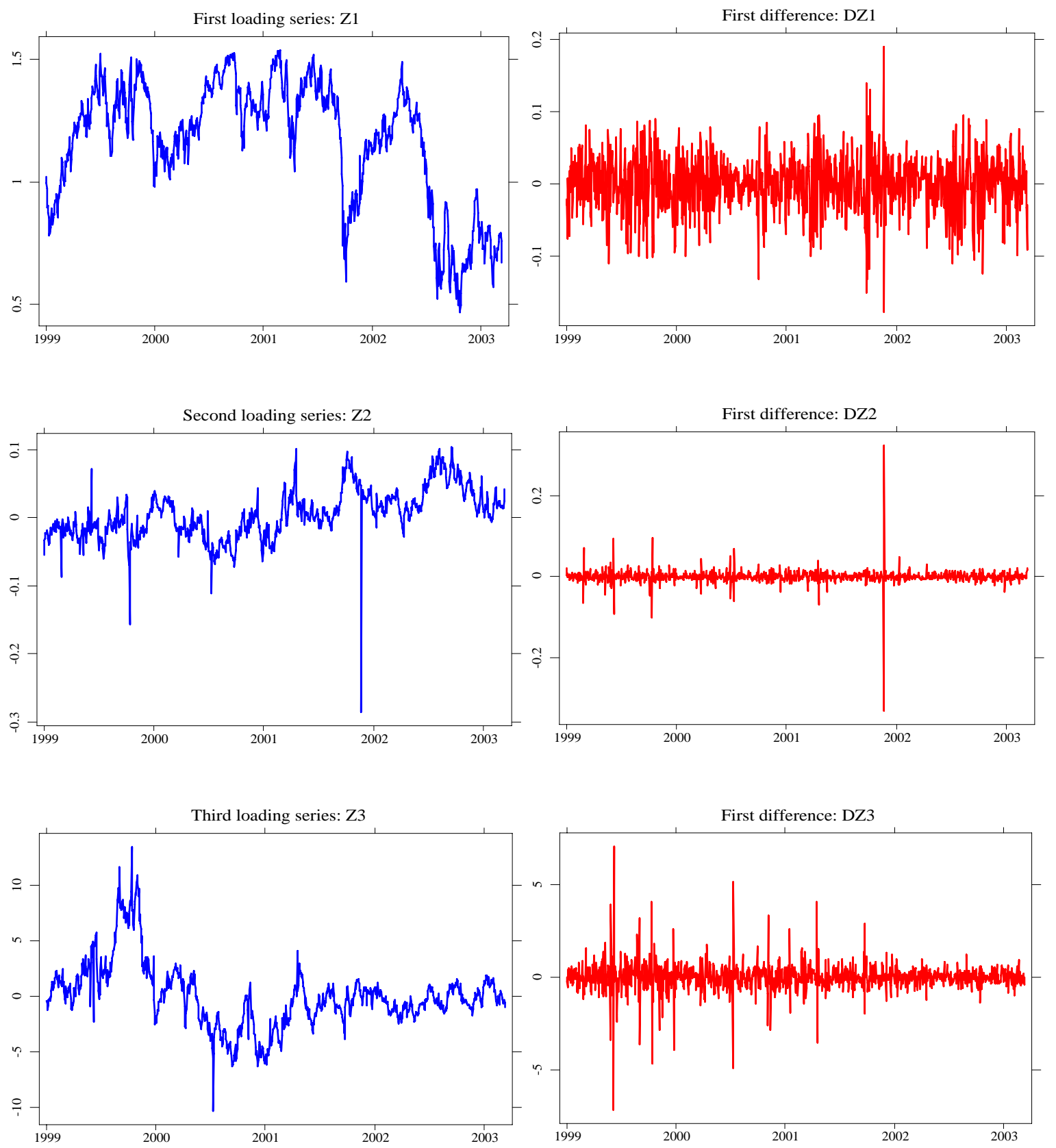

Figure 2: Factor Loading Time Series from Dynamic Semiparametric Model for Implied Volatility String Dynamics (left column) and first differences. 
Response to Cholesky One S.D. Innovations - 2 S.E.

Response of $\mathrm{Z} 1$ to $\mathrm{Z1}$

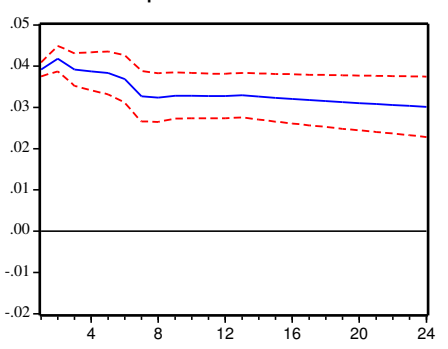

Response of Z2 to Z1

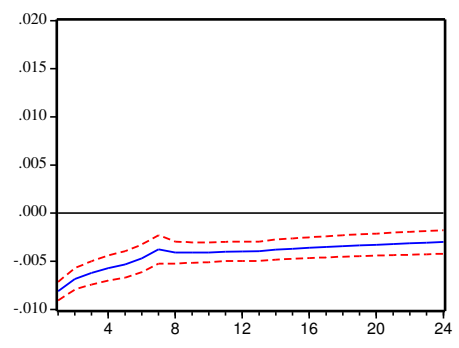

Response of Z3 to Z1

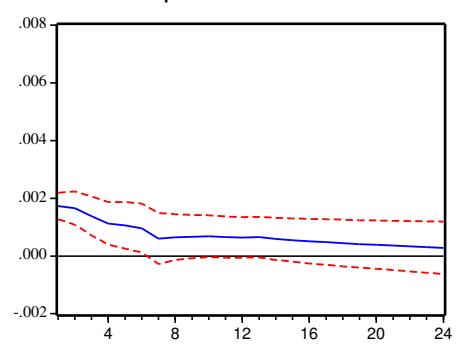

Response of Z1 to Z2

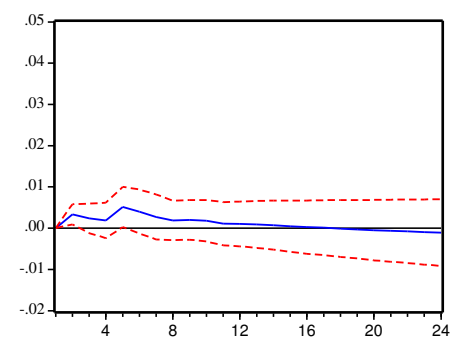

Response of Z2 to Z2

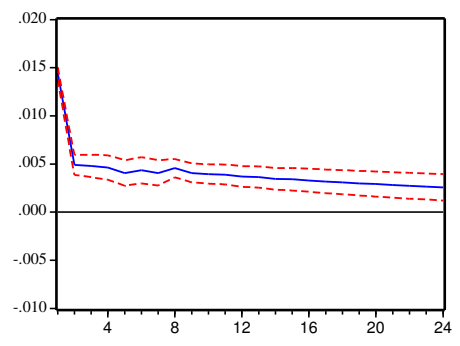

Response of Z3 to Z2

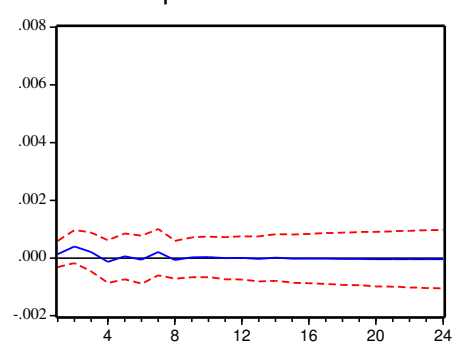

Response of $Z 1$ to $Z 3$

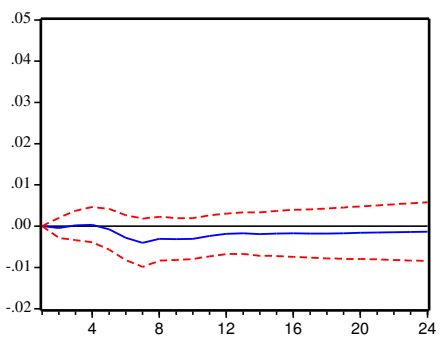

Response of Z2 to Z3

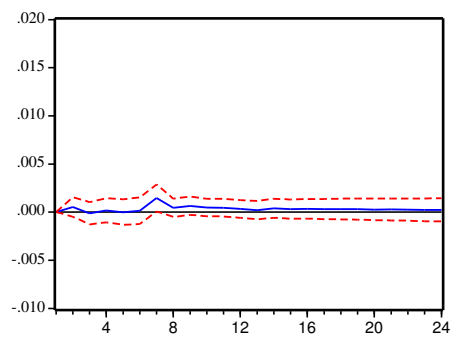

Response of Z3 to Z3

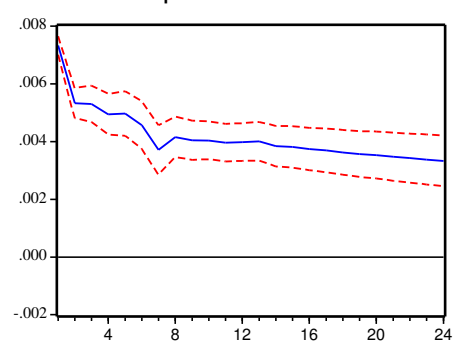

Figure 3: Impulse-Responses from a VAR(7) for $z_{t}=\left(z_{1 t}, z_{2 t}, z_{3 t}\right)^{\top}$. Sample: 1999/4/12003/2/25. 
Response of $Z 1$ to $Z 1$

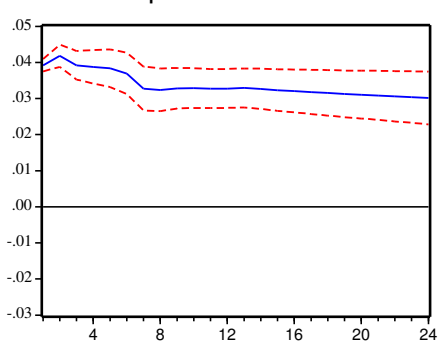

Response of Z2 to Z1

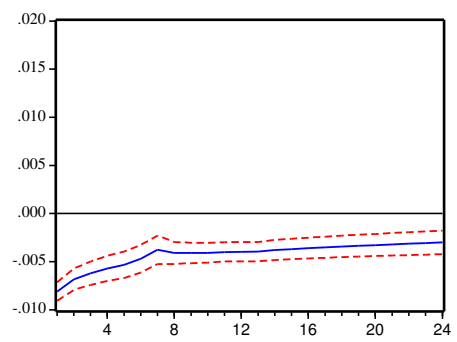

Response of Z3 to Z1

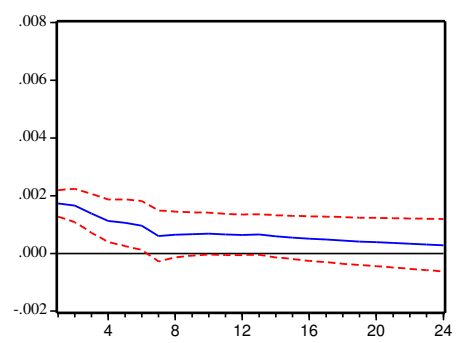

Response of Z1 to Z2

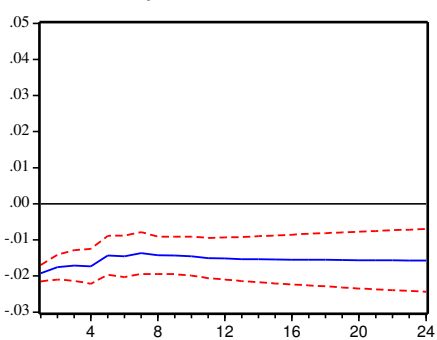

Response of Z2 to Z2

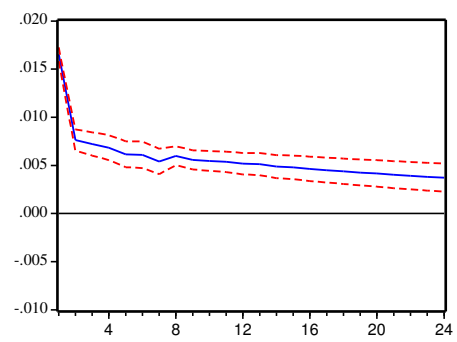

Response of Z3 to Z2

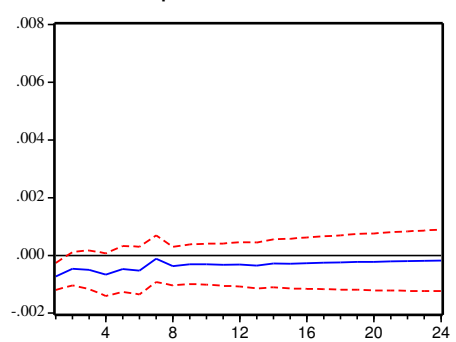

Response of $Z 1$ to $Z 3$

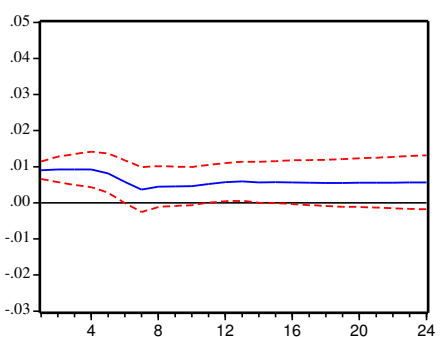

Response of Z2 to Z3

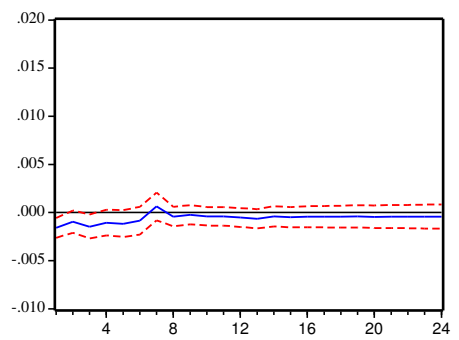

Response of Z3 to Z3

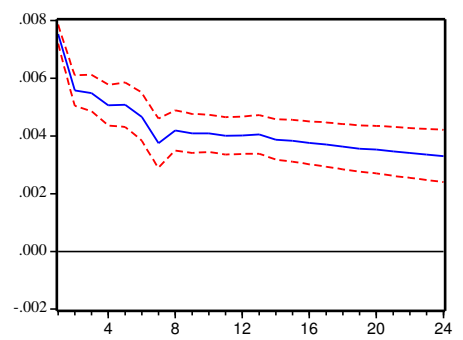

Figure 4: Generalized Impulse-Responses from a VAR(7) for $z_{t}=\left(z_{1 t}, z_{2 t}, z_{3 t}\right)^{\top}$. Sample: 1999/4/1-2003/2/25. 


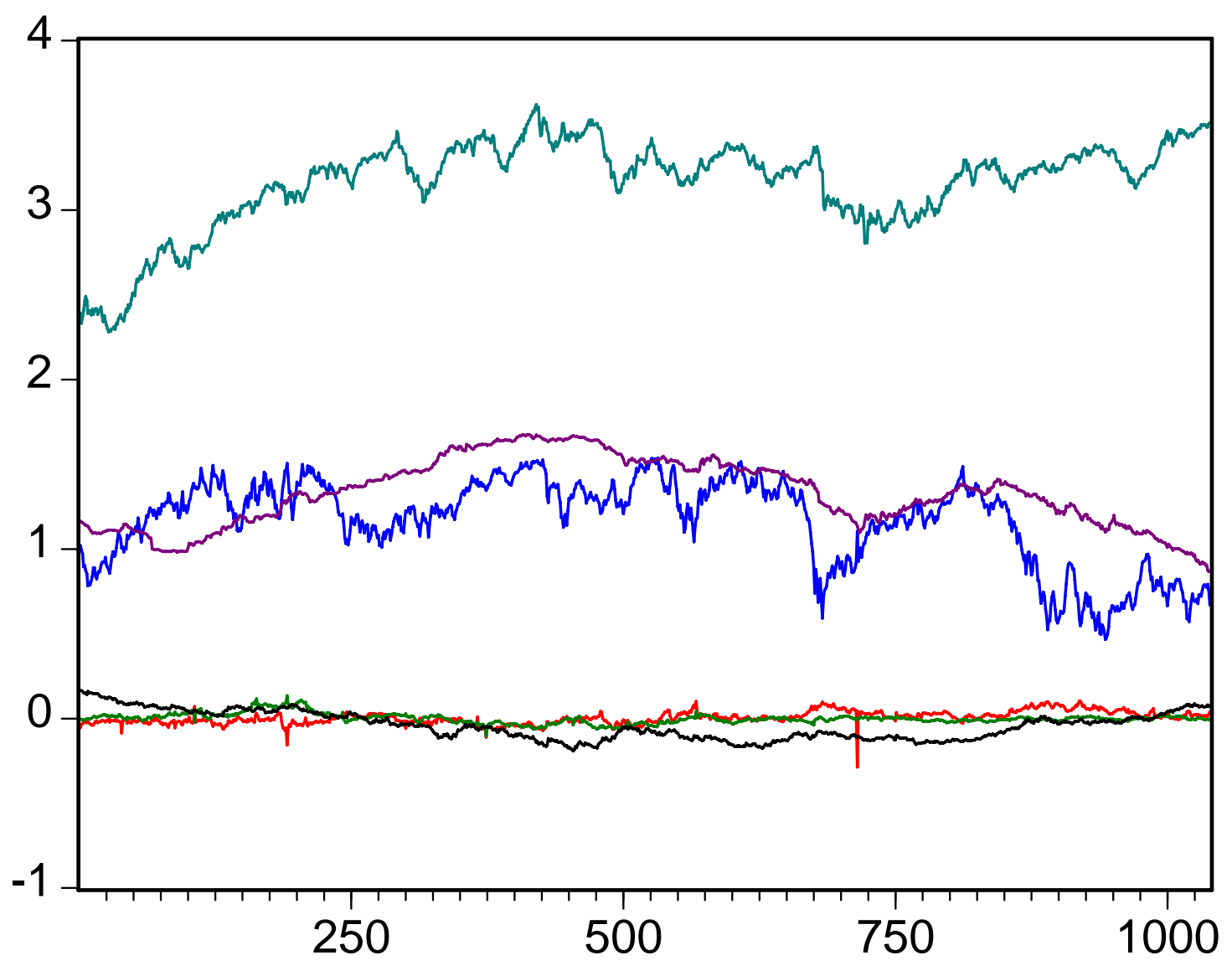

Figure 5: Time series plot of factor loadings and economic indicators for sample peroid: 1999/4/1-2003/2/25. $z_{1 t}$ (blue), $z_{2 t}$ (red), $z_{3 t}$ (green), log of US dollar per Euro exchange rate LEX(black), log of oil prices LPOIL(cyan), the 12-months interest rates $R 12 M($ purple). 
Response of LEX to Z1

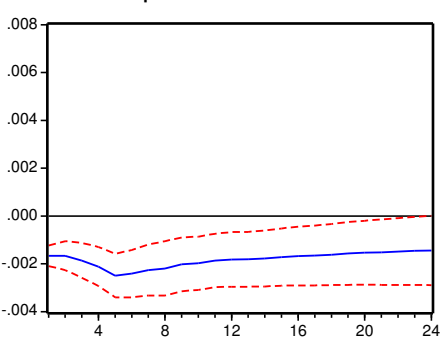

Response of LPOIL to Z1

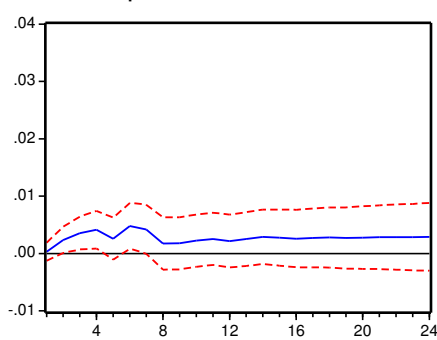

Response of R12M to Z1

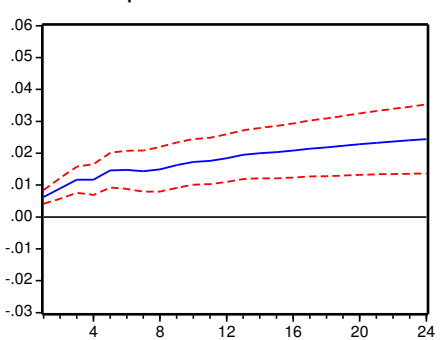

Response of LEX to Z2

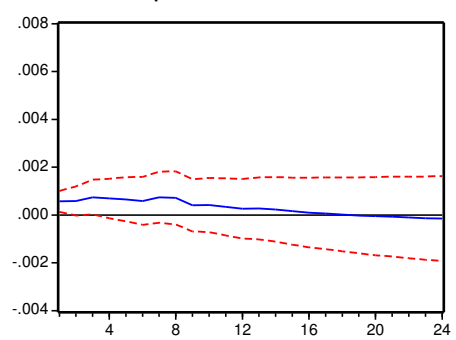

Response of LPOIL to Z2

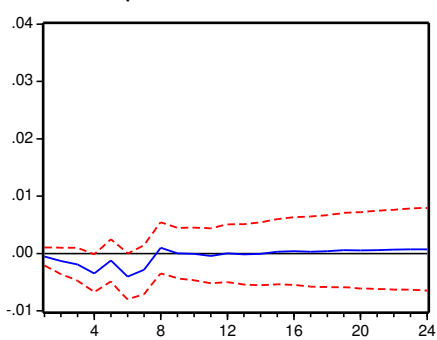

Response of R12M to Z2

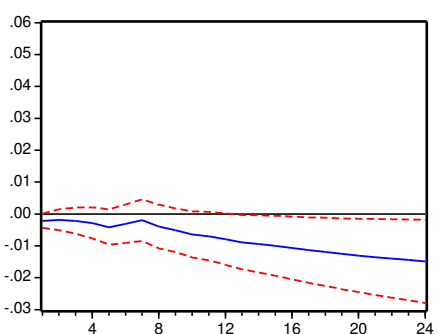

Response of LEX to Z3

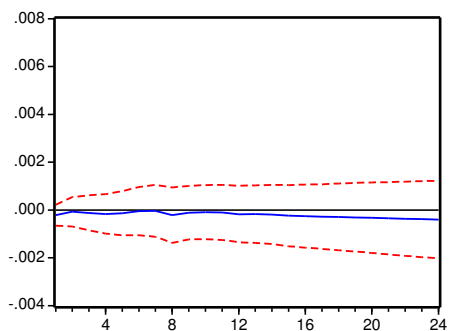

Response of LPOIL to Z3

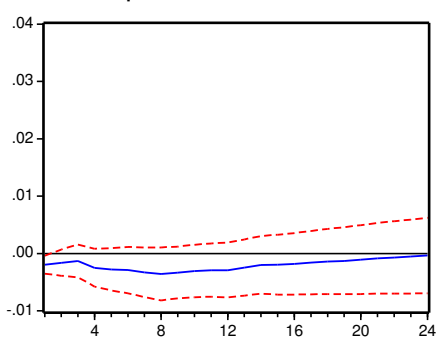

Response of R12M to Z3

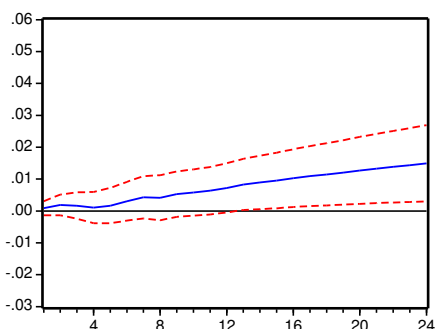

Figure 6: Responses of log of the US dollar per Euro exchange rate (LEX), log of oil prices (LPOIL) and the 12-month money market interest rate (R12M) to shocks in the factor loadings series: Generalized One S.D. \pm 2 S.E from a $\operatorname{VAR}(8)$ for $z_{t}=$ $\left(z_{1 t}, z_{2 t}, z_{3 t}, \text { LEX,LPOIL,R12M }\right)^{\top}$. Sample: 1999/4/1-2003/2/25. 

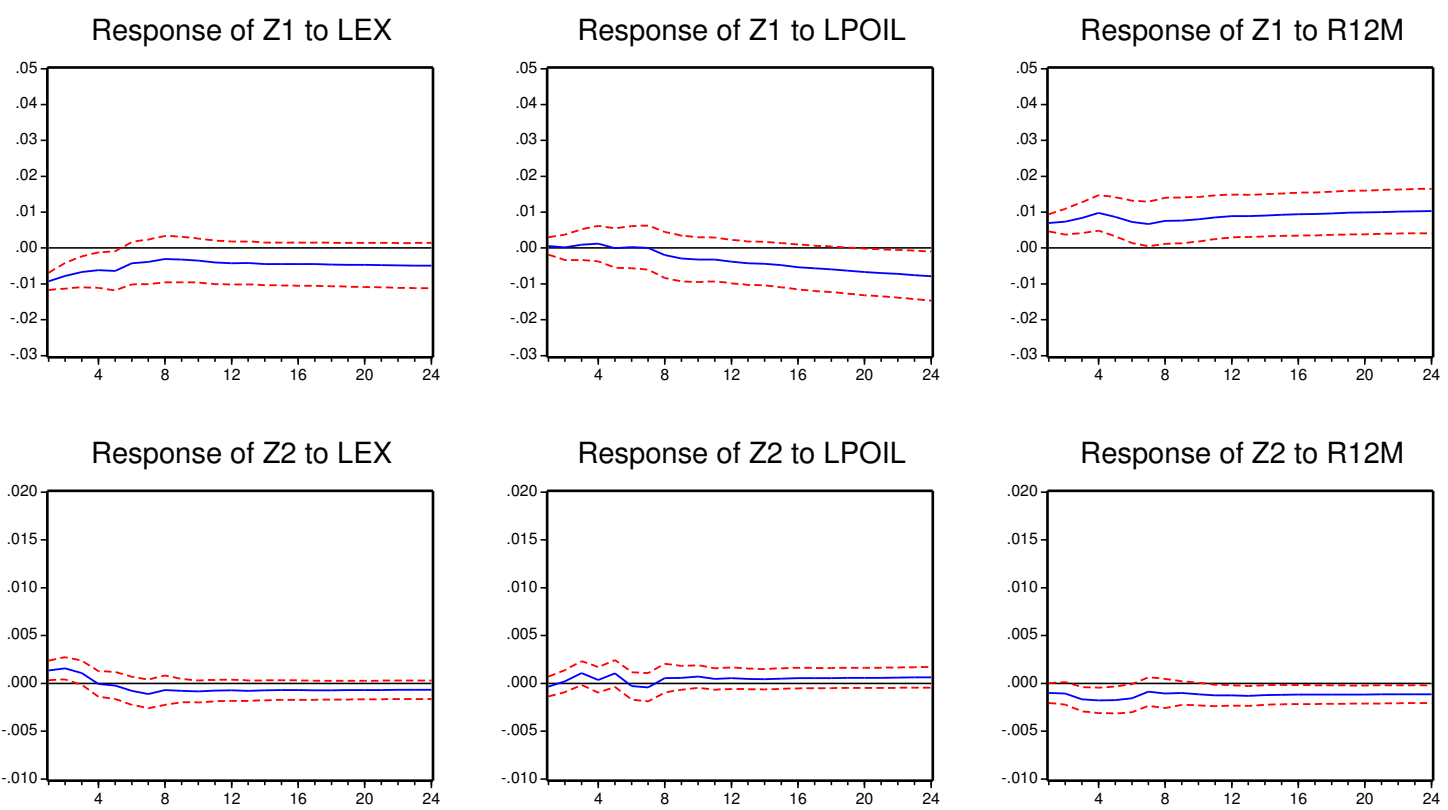

Response of Z3 to LEX

Response of Z3 to LPOIL
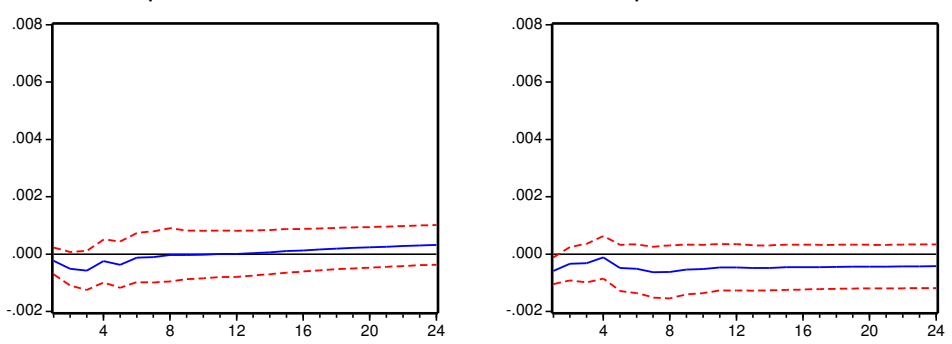

Response of Z3 to R12M

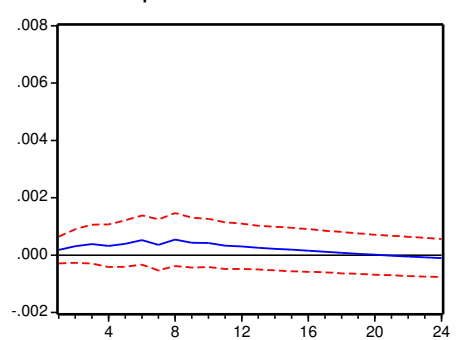

Figure 7: Responses in the factor loadings series to shocks in the log of the US dollar per Euro exchange rate (LEX), log of oil prices (LPOIL) and the 12-months money market interest rates (R12M): A Generalized One S.D. \pm 2 S.E from a VAR(8) for $z_{t}=$ $\left(z_{1 t}, z_{2 t}, z_{3 t}, \text { LEX,LPOIL,R12M }\right)^{\top}$. Sample: 1999/4/1-2003/2/25. 
Table 1: Unit Root Test Statistics

\begin{tabular}{ccccccccc}
\hline \hline Series & ADF-AIC & $\hat{p}$ & ADF-HQ & $\hat{p}$ & ERS-AIC & $\hat{b}$ & ERS-HQ & $\hat{b}$ \\
\hline$z_{t 1}$ & -1.982 & 6 & -2.241 & 2 & $3.787^{*}$ & 6 & $2.953^{* *}$ & 6 \\
& {$[0.295]$} & & {$[0.192]$} & & & & & \\
$\Delta z_{t 1}$ & $-15.199^{* * *}$ & 5 & $-23.582^{* * *}$ & 1 & $0.007^{* * *}$ & 5 & $0.075^{* * *}$ & 2 \\
& {$[0.000]$} & & {$[0.000]$} & & & & & \\
\hline$z_{t 2}$ & $-3.361^{* *}$ & 8 & $-4.219^{* * *}$ & 4 & 5.295 & 8 & $3.338^{*}$ & 4 \\
& {$[0.013]$} & & {$[0.001]$} & & & & & 7 \\
$\Delta z_{t 2}$ & $-15.646^{* * *}$ & 7 & $-15.646^{* * *}$ & 7 & $0.663^{* * *}$ & 7 & $0.663^{* * *}$ & \\
& {$[0.000]$} & & {$[0.000]$} & & & & & \\
\hline$z_{t 3}$ & $-2.874^{* *}$ & 7 & $-2.874^{* *}$ & 7 & $1.446^{* * *}$ & 7 & $1.446^{* * *}$ & \\
& {$[0.049]$} & & {$[0.049]$} & & & & & \\
$z_{t 3}$ & $-13.855^{* * *}$ & 6 & $-13.855^{* * *}$ & 6 & $0.005^{* * *}$ & 6 & $0.005^{* * *}$ & \\
\hline \hline
\end{tabular}

$A D F-A I C$ and $A D F-H Q$ refer to the ADF tests using the AIC and HQ criteria to estimate the lag length $p$ respectively. In case of ERS-AIC and ERS-SC the criteria used refer to the lag length $b$ chosen for the estimation regression of the autoregressive spectral density estimator. The critical values used for the ADF test are $-2.57(10 \%),-2.86(5 \%)$, and -3.44 (1\%) (Mackinnon, 1991). Elliot et al. (1996) state 4.48 (10\%), 3.26 $(5 \%)$ and $1.99(1 \%)$ as critical values for the ERS test. ${ }^{* * *}$ and ${ }^{* *}$ denote significance at the $1 \%, 5 \%$, and $10 \%$ level respectively. The $p$-values for the ADF tests are given in brackets below the test statistics. 
Table 2: Lag length suggested by information criteria

\begin{tabular}{ccccccc}
\hline \hline & \multicolumn{3}{c}{$1999 / 4 / 1-2003 / 2 / 25$} & \multicolumn{2}{c}{$1999-2001 / 7 / 31$} \\
Model & AIC & HQ & SC & AIC & HQ & SC \\
\hline$z_{t}$ & 7 & 3 & 2 & 3 & 2 & 2 \\
$\Delta z_{t}$ & 6 & 3 & 2 & 8 & 1 & 1 \\
\hline \hline
\end{tabular}

Table entries give the optimal number of lags determined using standard information criteria with a maximum lag order of $p_{\max }=$ 12. 
Table 3: $p$-values of diagnostic tests

\begin{tabular}{ccccccccc}
\hline \hline Model & Sample & $p$ & $\mathrm{Q}(20)$ & $\mathrm{LMF}(4)$ & $\mathrm{LMF}(8)$ & $\mathrm{LBJ}^{D H}$ & $\mathrm{LBJ}^{L}$ & $\mathrm{ARCH}(1)$ \\
\hline$z_{t}$ & full & 7 & 0.22 & 0.09 & 0.38 & 0.00 & 0.00 & 0.00 \\
$z_{t}$ & sub & 3 & 0.01 & 0.13 & 0.00 & 0.00 & 0.00 & 0.00 \\
$z_{t}$ & sub & 8 & 0.16 & 0.18 & 0.27 & 0.00 & 0.00 & 0.00 \\
\hline$\Delta z_{t}$ & full & 6 & 0.22 & 0.13 & 0.16 & 0.00 & 0.00 & 0.00 \\
$\Delta z_{t}$ & sub & 8 & 0.18 & 0.53 & 0.49 & 0.00 & 0.00 & 0.00 \\
\hline \hline
\end{tabular}

Diagnostic tests: full sample 1999/4/1-2003/2/25, sub-sample 1999-2001/7/31. Q(20) denotes an adjusted portmanteau test involving 20 autocorrelation matrices, $\operatorname{LMF}(4)$ and $\operatorname{LMF}(8)$ are $\mathrm{LM}$ tests for autocorrelation of order 4 and 8 . Two versions of multivariate Lomnicki-Jarque-Bera tests for nonnormality as described by Doornik and Hansen (1994) $\left(\mathrm{LJB}^{D H}\right)$ and Lütkepohl(1991) $\left(\mathrm{LJB}^{L}\right)$ and multivariate first order ARCH test are considered. All the tests are described in more detail in Lütkepohl(2004). Computations are performed with JMulTi, Version 5 (Lütkepohl and Krätzig, 2005). 
Table 4: Causality tests

\begin{tabular}{lr}
\hline \hline$H_{0}$ & Test result \\
\hline$z_{1} \nrightarrow z_{2}, z_{3}$ & $\mathrm{~F}(14,3072)=4.53[0.00]$ \\
$z_{2} \nrightarrow z_{1}, z_{3}$ & $\mathrm{~F}(14,3072)=1.66[0.06]$ \\
$z_{3} \nrightarrow z_{1}, z_{2}$ & $\mathrm{~F}(14,3072)=0.86[0.60]$ \\
$z_{3} \nrightarrow z_{1}$ & $\chi^{2}(7)=5.04[0.65]$ \\
$z_{3} \nrightarrow z_{2}$ & $\chi^{2}(7)=6.84[0.45]$ \\
$z_{1} \nrightarrow z_{3}$ & $\chi^{2}(7)=8.02[0.33]$ \\
$z_{2} \nrightarrow z_{3}$ & $\chi^{2}(7)=6.44[0.49]$ \\
$z_{1}, z_{2} \nrightarrow z_{3}$ & $\chi^{2}(14)=12.41[0.57]$ \\
\hline$z_{1} \stackrel{\text { inst. }}{\nrightarrow} z_{2}, z_{3}$ & $\chi^{2}(2)=224.5[0.00]$ \\
$z_{2} \stackrel{\text { inst. }}{\nrightarrow} z_{1}, z_{3}$ & $\chi^{2}(2)=202.5[0.00]$ \\
$z_{3} \stackrel{\text { inst. }}{\nrightarrow} z_{2}, z_{1}$ & $\chi^{2}(2)=57.7[0.00]$ \\
\hline \hline
\end{tabular}

Results are based on a model for $z_{t}$ using $p=7$ and data for the full sample period 1999/4/1-2003/2/25. $\nrightarrow$ denotes 'does not Granger cause'. $\stackrel{\text { inst. }}{\rightarrow}$ denotes 'does not contemporaneously cause'. $p$-values are given in square brackets. All the tests are described in more detail in Lütkepohl(2004). Computations are performed with JMulTi, Version 5 (Lütkepohl and Krätzig, 2005). 


\section{SFB 649 Discussion Paper Series 2006}

For a complete list of Discussion Papers published by the SFB 649, please visit http://sfb649.wiwi.hu-berlin.de.

001 "Calibration Risk for Exotic Options" by Kai Detlefsen and Wolfgang K. Härdle, January 2006.

002 "Calibration Design of Implied Volatility Surfaces" by Kai Detlefsen and Wolfgang K. Härdle, January 2006.

003 "On the Appropriateness of Inappropriate VaR Models" by Wolfgang Härdle, Zdeněk Hlávka and Gerhard Stahl, January 2006.

004 "Regional Labor Markets, Network Externalities and Migration: The Case of German Reunification" by Harald Uhlig, January/February 2006.

005 "British Interest Rate Convergence between the US and Europe: A Recursive Cointegration Analysis" by Enzo Weber, January 2006.

006 "A Combined Approach for Segment-Specific Analysis of Market Basket Data" by Yasemin Boztuğ and Thomas Reutterer, January 2006.

007 "Robust utility maximization in a stochastic factor model" by Daniel Hernández-Hernández and Alexander Schied, January 2006.

008 "Economic Growth of Agglomerations and Geographic Concentration of Industries - Evidence for Germany" by Kurt Geppert, Martin Gornig and Axel Werwatz, January 2006.

009 "Institutions, Bargaining Power and Labor Shares" by Benjamin Bental and Dominique Demougin, January 2006.

010 "Common Functional Principal Components" by Michal Benko, Wolfgang Härdle and Alois Kneip, Jauary 2006.

011 "VAR Modeling for Dynamic Semiparametric Factors of Volatility Strings" by Ralf Brüggemann, Wolfgang Härdle, Julius Mungo and Carsten Trenkler, February 2006. 